\title{
THE PLACE OF ULTRA-RADICAL SURGERY IN ADVANCED MALIGNANT DISEASE IN THE PELVIS
}

\author{
By J. B. Blaikley, F.R.C.S., F.R.C.O.G. \\ Director, Department of Obstetrics and Gynaecology, Guy's Hospital \\ Surgeon i/c Foint Cancer Clinic, Chelsea Hospital for Women and Royal Cancer Hospital
}

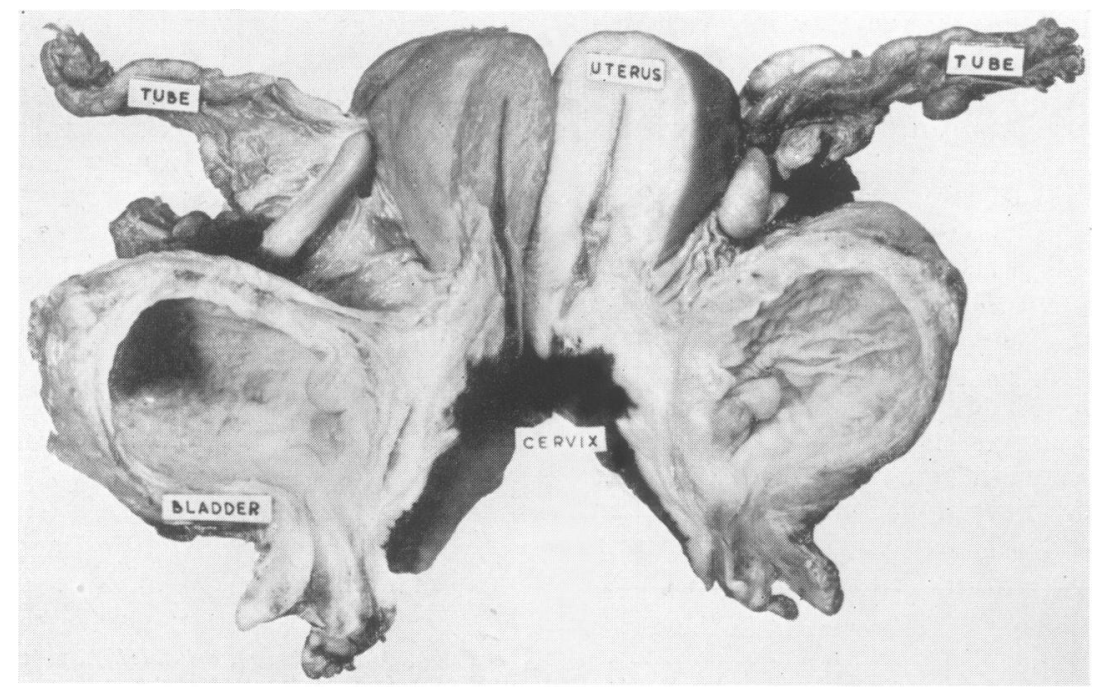

FIG. 1.-Anterior exenteration for carcinoma of the cervix with extension to left parametrium and base of bladder with obstruction of left ureter. The carcinoma was uncontrolled by radio-therapy completed two months previously. The patient is alive and well 4 years later.

The possibility of more extensive surgical procedures for the treatment of advanced malignant disease in the pelvis must have been in the minds of many for some years, but they have only become at all practicable recently with the development of modern anaesthesia, which causes the minimal disturbance of the patient, blood transfusion, and antibiotics. Even so the hazards are great. Brunschwig has been the great protagonist of ultraradical surgery and even he emphasizes that pelvic exenterations, as they are now usually called, are only on trial. None the less it is possible at this date to form some conclusions as to the results of these operations and to form an opinion as to their place in the treatment of malignant disease, in particular carcinoma of the cervix. Brunschwig has now been doing this type of surgery for seven and a half years and others of us for between four and fiveô years.

I will review the nature of the operations and음 indicate when they are applicable.

An anterior exteneration entails removal of the uterus, all or most of the vagina and sometimes then vulva, the bladder and terminal ureters, and all the ${ }^{\text {o }}$ pelvic cellular tissue together with the iliac and 0 obturator lymph nodes; it may mean removing a $\mathrm{N}_{\omega}^{N}$ good part of the levatores ani muscles. Whethero the operation can be completed by an abdominalo approach or whether it is necessary to combine ac perineal operation with the abdominal depends on $\stackrel{?}{?}$ whether the cancer is confined to the upper one- $\square$ third of the vagina or extends lower. Obviouslyo the severity of the operation is greater if it is necessary to adopt the latter procedure. When $\frac{\mathbb{\Phi}}{\alpha}$ 
the excision is completed, the pelvic walls and upper surface of the levatores ani are covered by smooth fascia of the muscles and the periosteum of the bone; the rectum, behind, hangs free of the pelvic wall, and the sacral plexus on either side shows plainly, while the iliac vessels stretch across the pelvic brim; (all the cellular tissues have been cleared away by blunt dissection with the fingers except perhaps for the final clearing of the rectum.) The ureters are implanted into the colon, usually $2 \frac{1}{2}$ inches and 5 inches below the junction of descending and pelvic parts; I implant them directly with the minimum of suturing and use the attached peritoneum to anchor them.

This operation is applicable to advanced carcinoma of the cervix which is not fixed to the pelvic wall, (a difficult point to determine sometimes) and in which the rectum is not involved, also to carcinoma of the vagina, to carcinoma of the urethra, and possibly carcinoma of the bladder, but this last is a matter for the genito-urinary surgeons. I will later give my views as to when this type of surgery should be used.

A total exenteration entails removal of the rectum along with the uterus, vagina, and bladder as above, and at the finish a wet colostomy is performed; this is best done at the umbilicus. Urine and faeces ultimately drain into a special bag attached to the skin. Again the operation may be entirely abdominal or part abdominal and part perineal. It is applicable to cancer of the cervix invading bladder and ureters and the rectum also. Advanced carcinoma of the vagina may be best treated in this way.

A posterior exenteration is really an abdominoperineal excision of the rectum together with excision of the uterus and vagina, and in the case of cancer of the cervix or upper vagina the pelvic lymph nodes must be removed. The bladder and ureters are left intact. The clearance of pelvic cellular tissue therefore is necessarily less complete than in the other two operations. It is applicable to carcinoma of the posterior vaginal wall, carcinoma of the posterior lip of the cervix spreading along the utero-sacrals or down the vagina with involvement of the rectum, and to carcinoma of the colon or rectum involving the uterus and vagina; I have done it for carcinoma of the colon with a large metastasis in the posterior vaginal wall, the patient is alive and well two and a half years later.

Posterior exenteration is in a different category from anterior and total exenteration simply because the ureters are not divided and transplanted into the colon. Transplantation of the ureters adds to the severity of the operation at the time, but above all it adds to the hazards afterwards, and of those

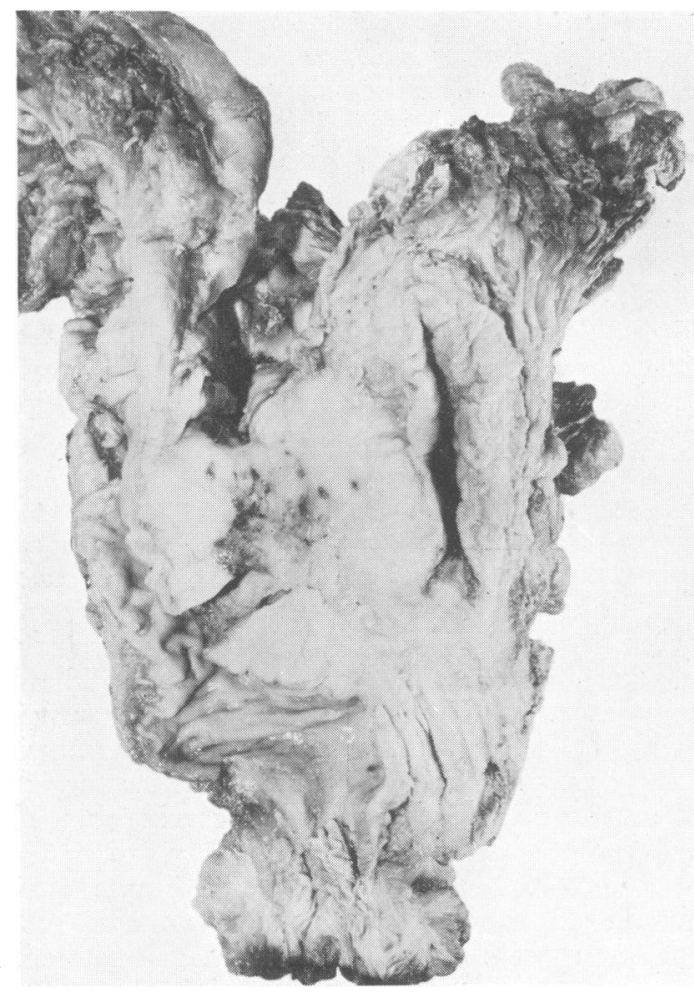

FIG. 2.-Total exenteration for recurrent carcinoma following carcinoma of the cervix treated by Wertheim's hysterectomy and subsequently radiotherapy which was unsuccessful in destroying this recurrence. The bladder is on the right and the rectum on the left. Both show extensive invasion. The patient is alive and well $2 \frac{1}{2}$ years later.

who survive the severe shock attendant to these operations many die from renal failure, usually consequent upon infection during the next few weeks or months, while some die from anuria or electrolyte imbalance during the next few days following operation. To avoid the risks of pyelonephritis surgeons have used skin nephrostomies, but these are uncomfortable and difficult to manage. Others recently have tried isolating a loop of intestine and transplanting the ureters into this loop, but this adds to the duration of the operation and it has been proved that the caecum or small intestine are unsatisfactory receptacles for urine owing to reabsorption of urea and sodium in particular.

What then is the place of these three types of operation? Are they to be used in primary treatment or only as a last resort when other measures such as radiotherapy have failed ? As I have said, posterior exenteration is easily the least severe, and is unquestionably the only treatment 
possible for carcinoma of the colon or rectum invading the uterus or vagina. It may be the best primary treatment for carcinoma of the posterior wall of the vagina since carcinoma of this organ does not respond very readily to radiotherapy, all the same I would ordinarily give the latter a trial because some cases do very well, and I would only operate if the response is poor or recurrence occurs. It is only very seldom that the operation is applicable in carcinoma of the cervis and never or very rarely I believe as primary treatment. It will be appreciated therefore that posterior exenteration is seldom performed by the gynaecologist, but has I believe a definite usefulness.

Anterior and posterior exenterations are very severe operations indeed, and it is no good attempting them on women who are in poor cundition; they must be under 65 years of age and physically fairly robust, and they must have stamina to help them over the often difficult convalescence. Even so the operative mortality is high, between 25 and 40 per cent. Brunschwig has recently reported on his results in the surgical treatment of carcinoma of the cervix obtained between September, 1947, and July, 1949; he performed 61 operations with I $_{5}$ deaths attributable to the operation; in most cases the operations were performed as primary treatment, but he states that there was only minimal selection on account of age and poor health. Of these $6 \mathrm{r}$ patients, 8 ( 13 per cent.) were alive and well between 4 years and 5 years ro months afterwards. Without knowing how many cases there were of recurrent carcinoma it is not possible to analyse the results further.

It is necessary to compare these results as far as one can with those of radiotherapy. In Scandinavia, over a series of several thousand cases of carcinoma of the cervix, it has been shown that it is possible to get nearly 50 per cent. 5 year cures in Stage II, 25 per cent. in Stage III, and ro per cent in Stage IV; very similar figures are shown by the Holt Radium Institute, Manchester, the Chelsea Hospital for Women and Royal Cancer Hospital Joint Clinic, and several other centres. If ultra-radical surgery is to be used as primary treatment in carcinoma of the cervix it has got to be possible to show better figures than these, and it would seem to me that this is not so at present and that it is most improbable that this will ever be the case in view of the primary mortality, and the subsequent deaths from renal failure, and in addition the often unsatisfactory extirpation of the cancer.

It seems to me therefore that these two operations should be employed in the treatment of advanced carcinoma of the cervix only in cases of radiotherapy failure; it is only in such cases that it becomes reasonable to take such big risks for such small results. I have taken this course and 3 my experience is therefore small at present; my first patient is alive and well after 4 years, but $ᄃ$ others have not done so well, and of 14 cases of $\overrightarrow{\vec{s}}$ carcinoma of the cervix operated upon (all previously treated by radiotherapy), of the remaining 4 alive, I has survived $2 \frac{1}{2}$ years and the other 3 only a few months. Way's figures for 33 cases $\overparen{\nabla}$ of total exenteration (he never does an anterior $\varrho$ operation) are similar, and so are Read's. In just के a few cases then, one case in 7 or 8 , it is possible to $\overrightarrow{0}$ usefully extend a woman's life by such surgery when all else has failed.

It would be a great advance if a really accurate $\frac{D}{\circ}$ method were evolved for determining early the $\frac{\mathscr{C}}{3}$ effect of radiotherapy in carcinoma of the cervix, it would then be possible to abandon this treat- O ment and undertake radical or ultra-radical surgery $\omega$ with the certainty that there was no alternative which would be both safer and as efficacious or more so. Glucksmann and Spear have attempted 0 to assess the response of tumours to radiotherapy by a differential cell count; after irradiation a $\vec{c}$ biopsy is made and the numbers of cells undergoing mitosis, resting, degenerating and showing $\overrightarrow{0}$ differentiation are estimated; in a favourab\& on response the numbers in the first two groups fas and in the last two rise; the response is unfavourabe in their opinion if the first two groups are not affected. However, it would appear that many showing an unfavourable response in fact do well

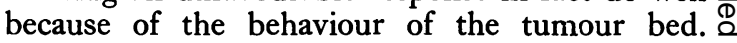
Graham claims that the response can be accurately $\overrightarrow{\overrightarrow{0}}$ predicted by an examination of 'smears' taken a 3 week after the first treatment with radium, and $\bar{P}$ if this claim is substantiated it will indeed be a considerable advance in helping the gynaecologists to know when to operate, and in advanced cases 3 . therefore when to undertake an exenteration, both $\dot{0}$ because the earlier the operation is performed the more chance of successful removal, and because $\delta$ following full irradiation with radium and deep $\mathrm{X}$-rays, the extensive fibrosis that occurs largely 윽 destroys all planes of cleavage. At the present time it is hard to tell whether a given cancer is going to respond or not, (some show considerable delay and then do well), and it is only after some weeks and even months that it becomes clear that $\tilde{N}$ the growth is not destroyed. In this time large $\underset{\omega}{ }$ malignant glands may become evident, obviously $\sigma$ adherent to the large vessels and pelvic wall soo that operation is by then quite useless. At the $\mathbb{\Phi}$ same time early exenteration can mean an unneces- $\stackrel{?}{+}$ sary operation with very likely a fatal result.

It remains to consider recent trends. Brun-

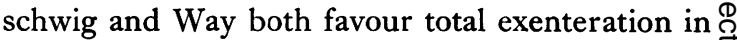
all cases as giving a maximum removal of possibly $\underset{\mathbb{Q}}{\mathbb{Q}}$ 
invaded tissue and also with the idea that there is less risk of ascending infection to the kidneys when continuous drainage is taking place into a bag. I favour anterior exenteration where possible because I think in suitable cases the prognosis is no worse qua recurrence of carcinoma or renal infection, and I believe most women can tolerate urine in the rectum more readily than a wet colostomy, even though they may be somewhat incontinent at night. A wet colostomy needs more clever management and more care than the average woman will give it, and it gets leaky and smelly very easily, at the same time I must admit I have a patient with a wet colostomy who does most things, and even plays the occasional game of golf (low handicap).

Way advocates the use of polythene catheters introduced into the ureters at the time of operation; he leaves the catheters draining into the colostomy bag for ten days and claims that they not only keep the wound dry but that the urine remains sterile. He reports no case of post-operative anuria and only a negligible rise in the blood urea at this time.
At the worst the patient dies quickly or after a few months, and usually she has had less pain and fewer other distressing symptoms than if she had not had the operation, and altogether life has been more tolerable; at the best she survives, feels well, and is capable of discharging her normal household duties and social engagements, and even of enjoying the theatre and outdoor exercise.

I will emphasize again however that exenterations, in my opinion, play only a very small part in the treatment of carcinoma of the cervix, and only in some 2 per cent. of cases is the operation applicable. It would be a great pity if ultraradical surgery comes to be used indiscriminately, and we must hope that one day other ways will be discovered for the cure or control of cancer of the cervix which make, such mutilating surgery quite unnecessary.

\section{BIBLIOGRAPHY}

BLAIKLEY, J. B., WAY, S., and READ, C. D. (1953), Proc. Roy. Soc. Med., 46, 753 .

BRUNSCHWIG, A. (1952), Cancer, 6, 980.

GRAHAM, R. (1953), F. Obstet. Gynaec., Brit. Emp., 60, 483 .

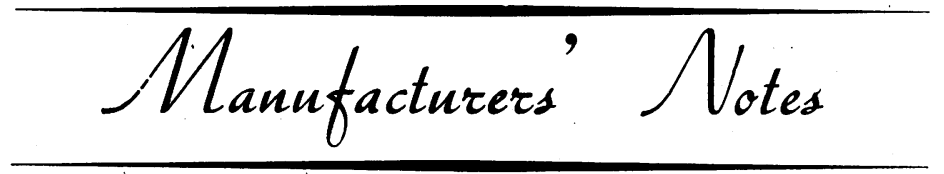

Pharmaceutical Specialities (May \& Baker) Ltd. announce the introduction of 'Diaginol' brand sodium acetrizoate, a water-soluble X-ray contrast medium containing 65.8 per cent. iodine and available as 30 per cent., 50 per cent. and 70 per cent. sterile aqueous solutions. This new medium has the advantage of producing side-effects similar in quality, but significantly less in severity and frequency than those encountered with diodone. Films obtained are comparable or superior in definition to those obtained with diodone and iodoxyl.

The indications for sodium acetrizoate solutions are similar to those for the established media. For these procedures, with the exception of cerebral angiography, 'Diaginol' 70 per cent. solution is the most suitable, though the 50 per cent. or even 30 per cent. may give adequate shadow where it is desired to minimize the risk of side-effects. No stronger solution than 30 per cent. should be used for cerebral angiography.

Presentation. 'Diaginol' brand sodium acetrizoate is available as 30 per cent. solution in 20 c.c. ampoules, 50 per cent. solution in 20 c.c. ampoules and 70 per cent. solution in 25 c.c. ampoules and 50 c c. bottles.

\section{' PENIDURAL' ALL-PURPOSE}

John Wyeth \& Brother Ltd., Clifton House, Euston Road, London, N.W.I, announce the introduction of 'Penidural' All-Purpose, a repository penicillin salt compound in aqueous suspension. Containing 300,000 units of potassium crystalline penicillin $G, 300,000$ units of procaine penicillin and 600,000 units of 'Penidural' (benzathine penicillin), it provides a high initial peak level that is maintained for an extensive period. Indicated in the treatment of most infections due to penicillin-sensitive organisms requiring high levels for the first day followed by prolonged serum concentration up to seven days with a single injection.

Available in dry powder form in 1.2 mega units, single-dose vials for intramuscular injection only. In the dry powder state it is stable for two years at $77^{\circ} \mathrm{F} .\left(25^{\circ} \mathrm{C}\right.$. $)$; after suspension with water for injection B.P., stable for seven days only if kept in a refrigerator. 